\title{
La sanità tra conservazione e necessità di cambiamento. Gli attori del cambiamento
}

\author{
Antonio Barracca \\ Medico, specialista in Nefrologia e Urologia, esperto di applicazioni per medici su piattaforma Android e Apple, Cagliari
}

\begin{abstract}
The health care system between tradition and need of change. The actors of this change
During these years we face changes to which we were not prepared, despite these changes have already been addressed elsewhere. Health care costs are becoming progressively higher and somewhat unsustainable because demographic changes are leading to a gradual aging of our population, due to low birth rates and longer life expectancy. Increasingly, longer life is lived in solitude. Therefore, the higher amounts of resources invested do not match the desired health outcomes.

The simultaneous aging of the population, the status of well-being that has eliminated many diseases, and a sedentary lifestyle that has brought others, have altogether increased the incidence of degenerative and cardiovascular diseases, as well as tumors, along with the conditions of obesity and diabetes. Consequently, more organs get sick simultaneously, for the same reasons, and for the same risk factors.

Therefore, we need to start aggregating the several fragmented medical cultures as to put man at the centre of care. Thus, we should recognize that the organizational models must adapt to these epidemiological changes, and that these changes involve not only the professions, doctors and nurses, but also the roles, powers, responsibilities, and the way we work, not as individual actors, but as protagonists in the care of sick patients with multiple organs involved, and no longer treating a single diseased organ.
\end{abstract}

Keywords: Organizational models, Medical skills, Assessments, Career progression

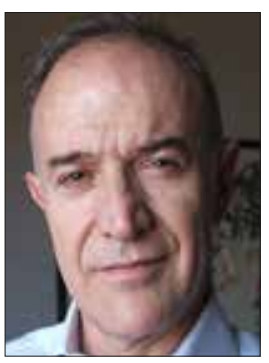

\section{Gli attori del cambiamento}

DIRIGENZA MEDICA E NUOVI PROTAGONISTI

\section{Direttore sanitario}

Penso che sia arrivato il momento per ridefinire, al di là delle normative vigenti, la figura del Direttore sanitario per dargli un ruolo non di factotum che controfir-

ma gli orari di servizio dei medici, ap-
prova la domanda di ferie, cerca di coprire turni scoperti nei

Antonio Barracca reparti, si occupa di trasferimenti di pazienti e di tante altre cose. Funzioni importanti che interessano i campi più dispara-

Accepted: January 30, 2015

Published online: February 23, 2015

Indirizzo per la corrispondenza:

Dr. Antonio Barracca

Medico, Specialista in Nefrologia e Urologia

Viale Merello, 74

09123 Cagliari

barraccaantonio@gmail.com ti, ma che, ad esempio, potrebbero essere in parte accorpate nella logistica, in una direzione alberghiera dell'ospedale, in una direzione autorevole del personale di assistenza. Forse bisognerebbe affidargli maggiormente compiti di gestione sanitaria ed essi sono veramente tanti.

Bisognerebbe cominciare a mettere a punto strumenti nuovi, ma già adottati in altre parti del mondo nell'ambito di quella che si chiama la Managed care. Per ogni paziente che entra in ospedale le direzioni sanitarie dovrebbero col tempo cominciare a determinare la durata teorica del ricovero, sulla base di parametri internazionali, accertandone quindi gli scostamenti che si verificano. Accanto a questa struttura sanitaria e in collaborazione è arrivato il momento di creare un team di economisti che si occupino non solo di economia aziendale, ma che comincino a studiare e mettere a punto database, sistemi integrati nei quali ciascun procedimento clinico diagnostico e terapeutico correlato alle cure al paziente sia analizzato. Per arrivare a una definizione di standard diagnostico-terapeutico a valenza regionale. Per essere più chiari: se prendiamo un qualsiasi capitolo della medicina e patologia umana, ad esempio, un paziente con focolaio di polmonite bilaterale esso deve avere una durata del ricovero prestabilita (ammettiamo 5 giorni), una serie di esami emato-chimici certi e validi per tutti i pazienti con la stessa patologia (fissi non 
variabili in relazione al medico, ma concordati con linee guida), una terapia con antibiotici o con una classe di essi anch'essa predeterminata, un impegno temporale di infermieri e medici conosciuto (durante il ricovero il medico curante non si occuperà di questo solo paziente, ma avrà altri impegni e quindi dobbiamo dare un costo in ore dedicate al lavoro del medico), tutti questi fattori di "produzione" devono avere un costo e nel complesso questo deve diventare un costo standard per tutto il servizio sanitario regionale che, per esser preso a base, deve essere confrontato con lo stesso costo ottenuto da altri servizi sanitari regionali. E alla fine, per ciascun paziente ricoverato e dimesso verrà calcolato il costo reale e lo scostamento dai costi standard dovrà essere giustificato dal Direttore di struttura. Resta l'altro aspetto, quello clinico della prognosi con i suoi esiti. Tutta questa immensa mole di dati dovrà col tempo essere creata per tutte le attività cliniche e per aver successo dovrà essere interfacciata con la cartella clinica digitale e con altri strumenti idonei.

Questo a mio parere è il futuro delle Direzioni e dei Direttori sanitari. Un compito importantissimo, indispensabile per avere un'idea dei costi e dei benefici e lo strumento principale per ridurre gli sprechi migliorando considerevolmente la clinica dei pazienti. Con uno strumento come questo potremmo decidere, ad esempio, di controllare meglio la durata delle degenze. Perché bisogna sapere che riducendo anche di un solo giorno la durata media delle degenze negli ospedali anche di una piccola regione si può determinare un risparmio di decine di milioni/anno. Ma non è parlando di macrostrutture che si modificano i conti e si tengono sotto controllo o si migliora la qualità delle cure. La chiave sta tutta nel funzionamento dei singoli sistemi. Tutto questo, però, non siamo stati ancora capaci di immaginarlo e di farlo.

Ma non dobbiamo scordare un punto focale, fondamentale. Dobbiamo capire, modellare e accompagnare il percorso di crescita e cambiamento della medicina ospedaliera e della sanità nel suo complesso. Chi dovrebbe occuparsi di tutte queste cose non ha tempo, né cultura, né sguardo rivolto al futuro. Dobbiamo anche noi introdurre nel nostro servizio sanitario a cominciare dai nostri ospedali più importanti una nuova figura, quella del Direttore scientifico. Perché se dovessimo portare avanti i suggerimenti sopraesposti resta da affrontare tutto il capitolo della qualità delle cure sanitarie, della loro attinenza alla Evidence Based Medicine (EBM), quali investimenti e indirizzi dobbiamo dare alla nostra sanità (quanta robotica pervaderà la sanità del prossimo futuro e cosa dobbiamo fare anche noi; quanta cardiochirurgia tradizionale verrà integrata e sostituita da pratiche endovascolari; quanta diagnostica radiologica verrà sostituita dalle macchine ecc). Le fila delle scelte e degli indirizzi che dobbiamo avviare negli svariati campi della medicina che cambia a velocità sostenuta non possono che essere tenute da un Direttore scientifico che avrà bisogno di una sua collocazione.

Ma questa importante e centrale struttura ha bisogno di figure di supporto professionali nuove, per noi sconosciute.
Economisti esperti di economia aziendale, informatici, esperti di statistica medica, epidemiologi, bioingegneri, operatori di sistemi informatici che inseriscano dati nei database clinici a supporto dei medici e altri ancora.

Basta pensare al gap che noi scontiamo. Il costo dei nostri sistemi amministrativi e di supporto nella sanità italiana è circa il $6 \%$ del costo totale sostenuto, contro il $36 \%$ degli Stati Uniti e in altri Paesi occidentali. Dobbiamo aumentare questi costi o meglio investimenti se vogliamo ridurre la spesa sanitaria.

Resta aperto il tema di cosa deve fare un ospedale, che qualità deve perseguire ogni Servizio sanitario regionale, come stare al passo con tutte le nuove scoperte, i nuovi strumenti tecnologici. Noi dobbiamo avere un'idea di sviluppo complessivo della qualità della pratica clinica consono alla EBM per impedire che ciascun Direttore di struttura complessa faccia scelte personali slegate dall'interesse generale.

La figura che dovrà occuparsi di questi aspetti strategici e fondamentali non potrà essere che una Direzione scientifica autorevole che si affianchi alla Direzione sanitaria e alla Direzione generale.

Non dobbiamo però essere preoccupati se questi cambiamenti nel tempo aggiungeranno nuove figure professionali, nuovi protagonisti della sanità. Lo scopo è quello di dare una nuova forma all'organizzazione, non quello di aumentarne i dipendenti. Questi passaggi, se fatti con criteri idonei porteranno a un miglior utilizzo di tutte le figure professionali, a una riduzione e a un miglior utilizzo dei medici, a un necessario aumento degli infermieri soprattutto per l'assistenza nel territorio che dovrà portare a una riduzione della spesa ospedaliera. Il sistema dovrà diventare più agile, dovrà consentire ai medici di dedicarsi all'assistenza e allo studio e lavorerà con meno medici e più personale di supporto. Lo scopo sarà quello di migliorare la qualità delle cure, ridurre i tempi di degenza con una più ampia dotazione di servizi e riuscire ad avere un rapporto fra costi e benefici verificabile.

\section{Direttore generale}

Dobbiamo avere a questo punto idee più precise sul ruolo, sulle capacità, su cosa chiediamo alla figura alla quale diamo la responsabilità dell'amministrazione della sanità pubblica, al Direttore generale. Spero siano passati i tempi in cui questi incarichi venivano assegnati esclusivamente da una parte politica indipendentemente dalle capacità e dal merito. Ciò non è più possibile. Chi si propone per questo incarico di grandissima responsabilità deve avere titoli specifici oltre che delle capacità riconosciute. E i titoli sono quelli che anche la legge ormai ritiene indispensabili. Avere conseguito una laurea, la maggior parte dei Direttori generali del Servizio sanitario nazionale (SSN) sono medici, aver frequentato una scuola, un corso universitario di Health Management ed avere esperienze importanti con titoli scientifici riconosciuti. Sapere amministrare una fabbrica della salute. Cosa fare allora? Chi aspira 
a questo ruolo, a questa carriera deve avere la giusta preparazione. E noi dobbiamo formare una nuova classe di amministratori preparati. La scelta per la politica sarebbe più facile perché ci sarà sempre qualcuno che potrà essere scelto per i suoi meriti e non solo per la sua appartenenza.

I compiti del Direttore generale sono delineati da norme, leggi e non dobbiamo reinventarli. Tutto ciò nella pratica di questi anni non è bastato; come non è bastato dire che uno dei loro impegni era quello di raggiungere il pareggio di bilancio della ASL o dell'ospedale che dirigevano. Tutto ciò, alla luce delle cose fin qui esposte è risultato minimale, risibile perché tutti sappiano che è stato necessario, e continua ad esserlo, ripianare gli sforamenti del bilancio delle ASL per centinaia di milioni/anno, che hanno determinato un deficit insostenibile per le casse pubbliche. La sanità invece si dovrebbe fare, realizzare solo con i soldi che la politica ogni anno decide di investire in questo settore. Non un euro in più può essere speso dai Direttori generali. Essi devono essere buoni amministratori e devono trovare le risorse necessarie all'interno del generoso finanziamento delle ASL. Faccio un esempio che tutti noi medici conosciamo. I reparti di Terapia intensiva degli ospedali hanno una gestione slegata dai Diagnosis Related Groups (DRG), per i loro costi elevati e per la gravità dei pazienti ricoverati. Capita spesso che un paziente dopo una fase iniziale di estrema gravità nella quale si impegnano molte risorse assistenziali, superata la stessa, questi bisogni si riducano, ma il paziente non può essere ancora trasferito in una degenza ordinaria. Servirebbe una degenza semi-intensiva che richiede meno personale, ridotta assistenza e costi di circa la metà di quelli sostenuti nella Terapia intensiva, per paziente. Pochi ospedali ne sono provvisti. Tutte le volte che qualcuno ha fatto la richiesta di avere una Terapia semi-intensiva la risposta è sempre stata la stessa. Servono risorse aggiuntive. Questo spiega come noi abbiamo bisogno di Direttori generali con capacità manageriali, con capacità di fare impresa. E allora il Direttore generale può creare una Terapia semi-intensiva, ed assumere se necessario il personale strettamente necessario. Sta facendo un investimento e deve avere pronto un conto economico perché la Terapia semi-intensiva la pagherà con i soldi che avrà risparmiato spostando i pazienti, che non hanno più bisogno, dalla Terapia intensiva a quella semi-intensiva. Se un giorno di terapia intensiva costa 3500 euro per paziente e se questo paziente starà ricoverato per 30 giorni, cioè fino alla sua dimissione per la mancanza di una Terapia semi-intensiva il costo del ricovero sarà stato di 105 mila euro. Al contrario la presenza di una Terapia semi-intensiva consentirà di spostarvi un paziente dopo la fase acuta con costi di circa 1500 euro/giorno e un possibile risparmio di migliaia di euro per paziente. In questo modo si dà un servizio migliore e vengono coperti i costi di questo nuovo servizio senza gravare sulla collettività. Questo è il ruolo di un amministratore della sanità. Perché è troppo facile spendere e far debito.

II Direttore generale, quindi, è responsabile dell'organizzazione, dello sviluppo, della qualità della sanità erogata. Per questo motivo è necessario dargli compiti precisi, non di programmazione sanitaria, come da qualche parte sta succedendo, laddove l'accorpamento delle ASL e la riduzione del loro numero ha portato i Direttori generali ad essere investiti di questo ruolo. Non era questo il compito del Direttore generale. Semmai la parte pubblica avrebbe dovuto chiedere spiegazioni del perché quel Direttore generale non fosse stato capace di aumentare il tasso di occupazione offrendo prestazioni sanitarie migliori ai propri cittadini che proprio per questa carenza avevano scelto di farsi curare in altre ASL.

Con gli strumenti che ho cercato di esporre tutto dovrà esser visto in una luce diversa. II Direttore generale sarà responsabile della gestione complessiva della ASL e dovrà essere capace di amministrare il budget affidatogli, migliorare la qualità del servizio. Ma non basta. Uno dei doveri principali sarà quello di aumentare la produttività del nostro servizio sanitario, a cominciare dagli ospedali, per continuare con la medicina del territorio. Questa bassa produttività che noi non sappiamo misurare, ma che non affrontiamo neppure da lontano è frutto della mancanza di standard, di obiettivi. Tutto è affidato a ciascun servizio, a ciascun Direttore di struttura. Sono loro i padroni. La politica con la Managed care se ne deve riappropriare. Per far questo il Direttore generale dovrà mettere a punto, usare tutti quegli strumenti, tutte quelle figure professionali, tutti i modelli organizzativi necessari che in parte ho delineato e che devono essere fatti funzionare. Ma c'è un aspetto troppo a lungo trascurato. Ricreare un senso di appartenenza al nostro servizio sanitario. Ridare a ciascuno il senso del ruolo che occupa e farlo attraverso il riconoscimento del merito. I medici, a cadenza periodica non possono aspettare la nomina del nuovo Direttore generale per sapere come muoversi, per ingraziarselo e ottenere le risorse necessarie al reparto. Deve essere chiaro che un ospedale, un servizio ospedaliero è di prestigio non per il Direttore generale di turno, ma per la preparazione dei medici e del personale che vi lavorano. In mancanza di questa cultura assistiamo a Direttori generali che si vantano di aver acquistato una TAC di ultima generazione che dà lustro all'ospedale, mentre dovrebbero comunicare ai cittadini che in quell'ospedale non solo grazie alla TAC, ma soprattutto alla sapienza dei medici la qualità generale delle cure è progressivamente migliorata.

Insomma i Direttori generali devono tornare ad essere dei gregari che lavorano perché il sistema sanitario funzioni bene e consenta ai cittadini di ricevere una sanità di buon livello per le capacità di medici e infermieri.

Per dare un senso concreto a tutte le cose dette è necessario che ogni Direttore generale prenda l'impegno di presentare una sintesi annuale dell'attività di un ospedale, di una ASL per farci conoscere lo stato del nostro servizio sanitario e per avvicinarlo ai cittadini.

Per raggiungere questi obiettivi la politica è incaricata di fare delle scelte nella sanità. Deve decidere quanti soldi la collettività deve investire in salute; quali farmaci e quali prestazioni, quali strutture possiamo permetterci, rispetto 
a quanto abbiamo deciso di spendere in salute. Sbaglierebbe se pensasse che il suo ruolo sia quello di decidere se servono più o meno ASL perché i costi che sosteniamo non dipendono dal numero, ma da come esse funzionano, da quale controllo la parte pubblica pretende di avere di queste repubbliche chiamate ASL. Se noi dovessimo accorpare le attuali ASL, senza i cambiamenti sottolineati, avremmo paradossalmente un aumento degli sprechi e un peggioramento della qualità. Se noi pensassimo inoltre di non vedere il servizio sanitario come un tutt'uno, ma come una parte che si occupa di salute nel territorio, un'altra dell'assistenza ospedaliera, una ancora delle emergenze e cosi via, avremmo una conflittualità continua perché gli ospedali non vor- rebbero ridurre i loro posti letto, la medicina del territorio vorrebbe i soldi degli ospedali per curare i loro pazienti e cosi via.

Serve una visione globale di tutti i servizi che funzionino in modo dipartimentale. La politica deve decidere che risultati di salute vuole avere. II sistema deve avere i controlli e l'organizzazione che ho cercato di delineare e sui quali le discussioni e le decisioni devono essere aperte al confronto.

\section{Disclosures}

Financial support: No financial support was received for this submission.

Conflict of interest: The author has no conflict of interest. 